\title{
End-Effector Airbags to Accelerate Human-Robot Collaboration
}

\author{
Roman Weitschat, Jörn Vogel, Sophie Lantermann, and Hannes Höppner
}

\begin{abstract}
A fundamental problem in human-robot collaboration is to ensure safety for humans being located in the workspace of the robot. Several new robots, referred to as collaborative robots, are pushing into the market. Most of these so-called co-bots have similar properties. They are small, lightweight and designed with big roundings to ensure safety in the case of a collision with a human. Equipped with torque sensors, external torque observers, tactile skins, etc., they are able to stop the robot when an emergency occurs. While developing more and more co-bots, the main focus lies on the robot itself. But to make a robot deployable, a special tool for a defined task is needed. These tools are often sharp-edged and dangerous in case of a collision with a human. In this paper we present a new safety module for robots to ensure safety for different tools in collaborative tasks. This module, filled with air pressure during the robot motion, covers mounted tools and carried workpieces. In case of a non or very slow moving robot, the safety module is able to pull back and the tool is uncovered. In our experiments we found out that we can increase the velocity up to $1 \mathrm{~m} / \mathrm{s}$ while satisfying the requirements of the ISO/TS 15066 and retain the full functionality of the tool.
\end{abstract}

\section{INTRODUCTION}

Human-robot collaboration is currently one of the main topics in the robotics community. Regarding industrial applications the demand on fenceless robot cells with collaborative and assistive robots is steadily growing. There are different types of human-robot collaboration. One type is a robot which is separated by light sensors, vision-based workspace observer [1] or for example pressure sensor mats [2], that trigger a robot to stop if a human enters the robot workspace during an autonomous motion. In case a human steps into the workspace the robot stops immediately to prevent any dangerous situation. Nevertheless, the human is able to interact with the robot in an idle state or put it into a handguided mode. The danger of a severe injury is potentially low. Another type are autonomous robots without any boundaries or safety sensors around the workspace. Humans are able to enter the workspace of the robot while it is moving and the risk to collide with the robot is highly probable. Robots such as the DLR lightweight robot LWR III [3] are able to react to a collision by detecting external torques, and therefore, they are able to safely collaborate with humans under specific conditions (see Fig. 1). For collaborative requirements the ISO 10218 [4] was introduced to define particular conditions to allow human-robot collaboration. Recently an extension was defined to specify "safety requirements for collaborative industrial robot systems and the work environment" [5] which defines conditions for collaborative

All authors are with Robotics and Mechatronics Center, DLR - German Aerospace Center, Wessling, Germany, contact: roman.weitschat@dlr.de

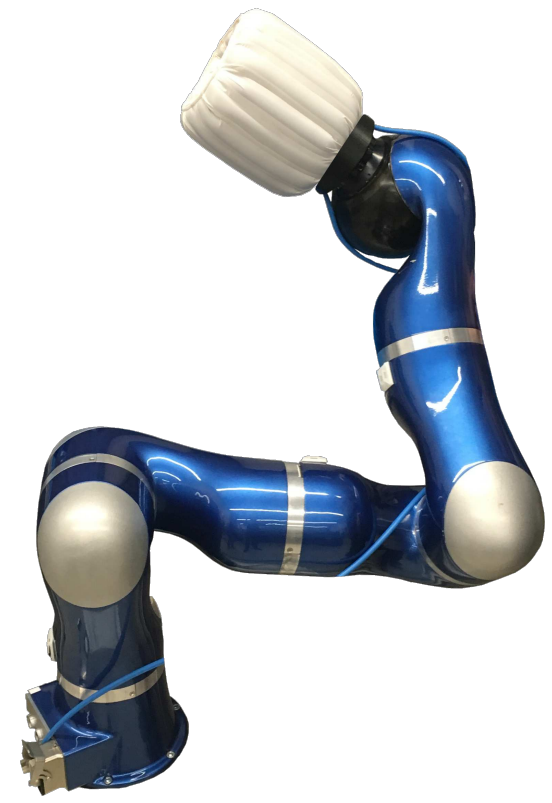

Fig. 1: The DLR Light-Weight-Robot - a sensitive torque controlled robot for safe human-robot collaboration equipped with a new module to ensure safety for all sort of tools.

robots: the technical specification ISO/TS 15066:2016. The key content of the technical specification describes maximum contact forces and peak pressures for a quasi-static and a transient contact.

Heretofore, fundamental work was done by the robotics community that executed many experiments and crash tests and analyzed the resulting effects to a human. Scientific pioneering work was done by Yamada et al. who introduced human pain tolerance as a criterion for safe robot impact behavior [6]. Thereupon, a significant work was done by Haddadin et al. who analyzed injuries with different robots considering the severity of injuries to a human caused by a collision with a robot [7], [8], [9], [10]. The resulting information exhibits that the severity of an injury depends on the mass, geometry and velocity of the colliding object [11].

To prevent severe injuries the safety community developed a wide variety of solutions for example actuation mechanisms [12], [13], collision avoidance control schemes [14], [15] and vision/sensor-based human detection and prediction [16]. However, all these technologies are not yet or only partially allowed for industrial applications. Therefore, we focus on hardware solutions to prevent danger to humans for accelerating human-robot collaboration technologies. There exist different solutions like using capacitive sensors [17] or 

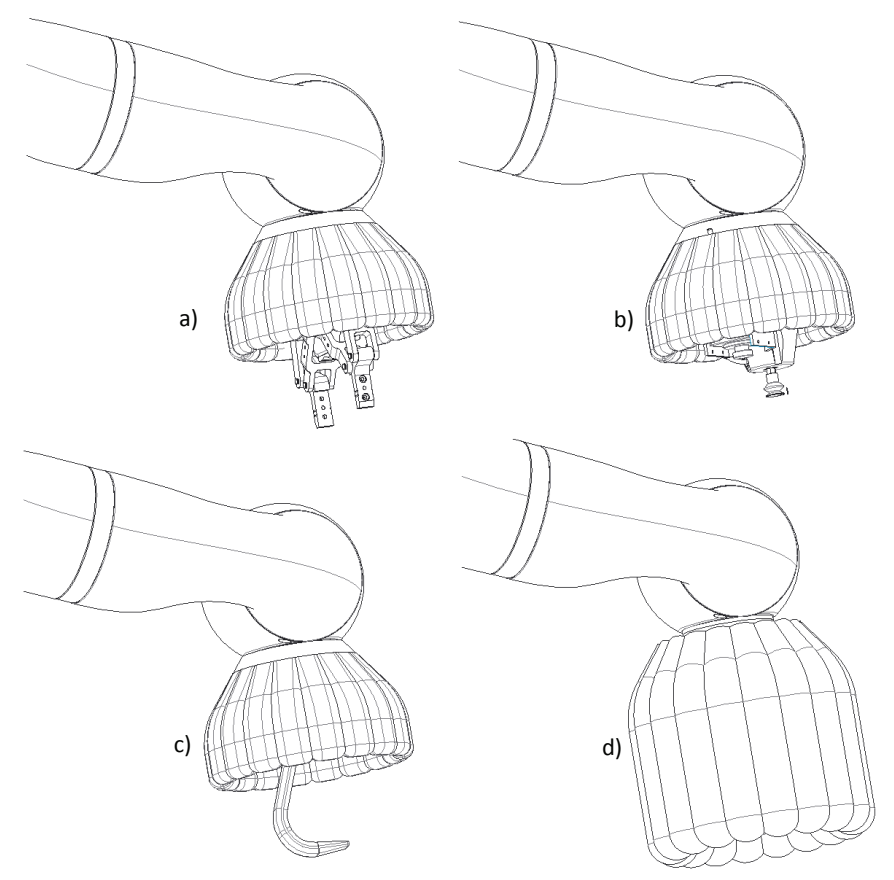

Fig. 2: Functionality of the DLR safety module; a) deflated module with a standard two-finger gripper; b) deflated module with a vacuum gripper and camera; $c$ ) deflated module for random type of, for example, 3D-printed tools, hooks etc. (similar used in a known automobile manufacturing); d) inflated module covers a), b) and c) completely and ensures a safe and fast motion without harming a human.

protective skins as cushion at the robot [18], [19]. These solutions are made to cover the robot structure but only partially the robot tool. Using protective skins for tools has one distinct disadvantage. It is highly probable that they influence the functionality of the tool. Recently, Shunk presented a gripper called Co-Act equipped with capacitive sensors to stop the robot in spatial proximity [20] which is quite a plausible solution for the problem of unsafe tools. However, the product was a case study and is only related to grippers from Schunk which leads to a deficit in flexibility for the manufacturer.

In this paper we present a new method to cover potentially unsafe tools of all sorts to enable economic human-robot collaboration and to increase performance while satisfying mandatory regulations. The paper is organized as follows. In Sec. II we describe the approach to cover unsafe tools for ensuring safe motions in collaborative tasks. Sec. III describes the experiments and analyzes the results by using impact force measurements and Fujifilm prescale pressure measurement film. Section IV concludes the paper and gives an outlook to future work.

\section{APPROACH}

As mentioned above, one big issue of bringing robots into collaborative or interactive tasks are unsafe tools with sharp edges. In this section we present a new approach which enables safe human-robot collaboration in industrial tasks by covering these edges. The requirements to a safety system for tools are basically that the tool is covered and safe during a fast motion of the robot. Furthermore, there should be no restrictions for the tool when it is needed for gripping or manipulating an object. To achieve this, a mechanism is needed that is able to extend around the tool in case of an insecure situation, which basically always occurs when a robot moves autonomously. Additionally the mechanism has to be able to retract itself when the full functionality of the tool is required.

This is the motivation of the paper at hand. We developed an airbag-like safety-module which builds a cushion between the robotic end-effector and the human in case of a collision (see Fig. 1). The main idea is, that the airbag does not trigger at an approaching danger but is rather always inflated during unsafe motions of the robot. This leads to an intrinsically safe motion with an unsafe end-effector. The inflation is realized by air pressure which is commonly existent in every industrial production.

The second and very important feature of the safety module is that it is able to deflate when the robot is standing still or moving very slow and safe. The deflation-depicted in Fig. 2 a), b) and c) —in combination with elastic bands or springs allows to pull the covering away and therefore provides open access to the tool.

The selected material is Nylon since it is an established and well-proven material for airbags as used in cars and bicycle helmets, for example. The design is a special construct of chambers to reduce the overall volume and brings it into a desired shape such that the tool is fully covered. The reduction of volume is an important aspect because the smaller the volume the faster is the inflation and deflation. This is due to the air flow in the supply pipe which has a limited diameter when it is led through the robot. To ensure fast cycle times it is necessary to optimize the inflation time of the safety module. But it is recommended not to inflate explosively, because of potential injuries to humans or that the tool drops unknown gripped objects, even in idle state.

As mentioned above using foam as safety cushion does not allow to free the tool. With the developed safety module we are able to evacuate the airbag and allow to pull back the covering. The pull back can be done by elastic bands or springs. However, it is equally important to deflate and pull back the airbag. The difference for deflating is that it can be done as fast as possible because it cannot emerge a danger by deflating the airbag.

As one can see in Fig. 2, the safety module is not depending on a special tool. This means it is possible to rapidly recreate new tools, for example by 3D-printing and covering it with the safety module. The size of the airbag can also be customized very easily and enables fast set-up times. However, the robot should fit the requirements of HRC, e.g. collision detection.

Another advantage of the developed safety module is that it covers more than only the tool. The customization can also depend on the carried object of the robot. The form can be adjusted so as to cover the object, too, and therefore a safe motion with carried object is possible, as well. 

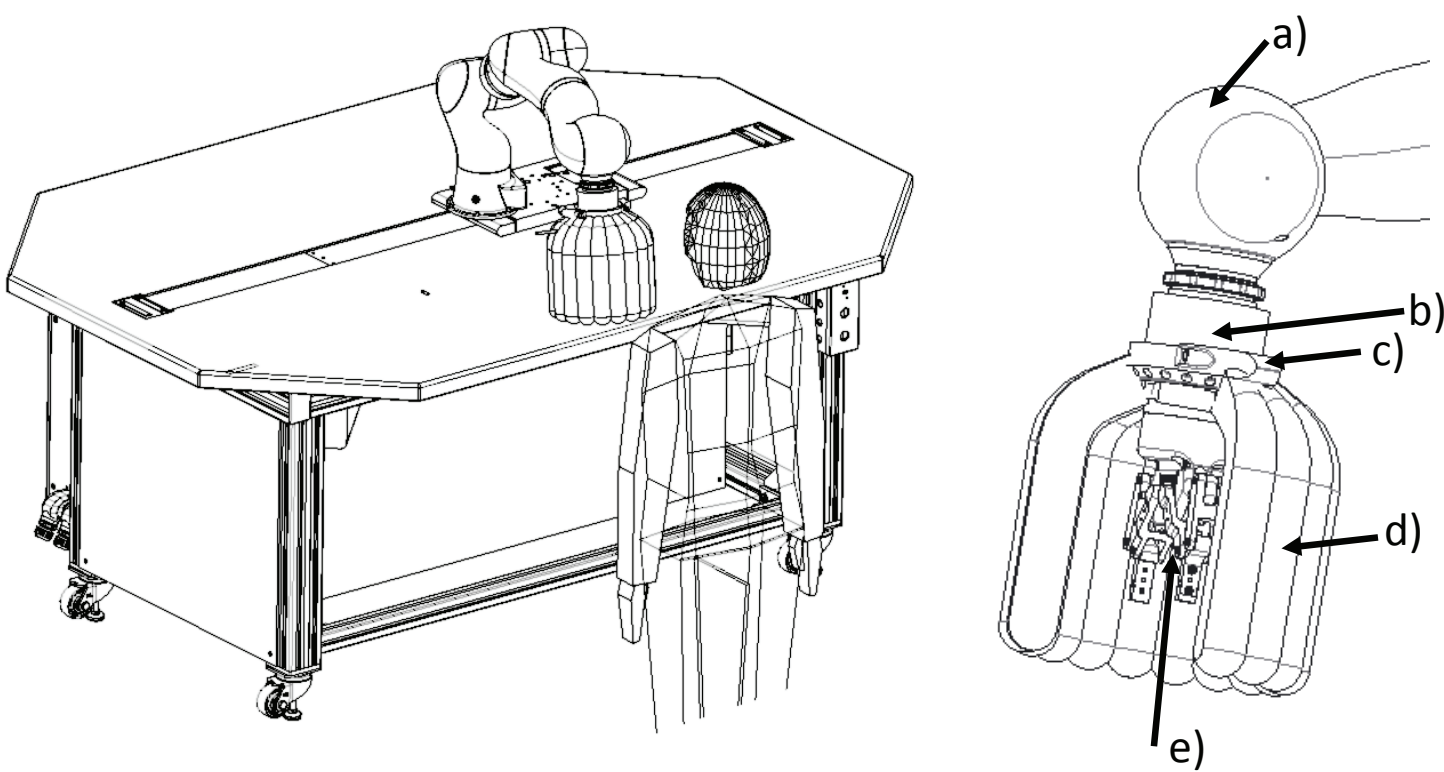

Fig. 3: a) DLR lightweight robot LWR III, b) JR3 six-DoF force-torque sensor, c) valve and connection to airbag flange, d) airbag, filled with air pressure e) standard two-finger gripper

In case a human is clamped by the robot and is not able to free himself because the joint-brakes are activated, we are able to deflate the airbag, for example after a defined time of the collision, and the human is finally free.

The measurements revealed an inflation time of approximately $0.5 \mathrm{sec}$. In the experiments the inflation was triggered right before the start of the motion without a delay. Less than a second, currently $0.8 \mathrm{sec}$., is needed to deflate the safety module. Nevertheless, the deflation time has the potential to be further reduced. The functional safety can be achieved by sensing the current pressure.

\section{EXPERIMENTS}

In this section we describe the experimental setup we used for collision experiments with a crash-test dummy and the DLR lightweight robot LWR III. Then, we compare collisions without an airbag covering the tool and an inflated airbag. Therefore, we analyze the arising forces depending on different velocities during the impact. Finally, we analyze the impacts with Fujifilm prescale pressure measurement film [21].

\section{A. Experimental setup}

The experimental setup is depicted in Fig. 3 We chose to use an exemplary setup which is similar to a collaborative workbench which could be used someday in real industrial scenarios. The workbench is equipped with an eight degrees of freedom (DoF) robotic system. The sevenDoF DLR light-weight robot is mounted on a linear axis to extend the workspace of the robot. The weight of the robot is about $14 \mathrm{~kg}$. The linear axis is drive-belt-driven from Bosch Rexroth with a position sensor at the driven end and a link-sided position sensor to ensure redundancy in the velocity measurement. As tool a common two-finger gripper is used. The system with eight DoF is position-controlled during the experiments to ensure comparable motions for the experiments with and without the safety module.

Between the end-effector with the attached safety module and the robot TCP a six-DoF force-torque sensor (FTS) is mounted. The sensor is a JR3 FTS which is used to measure impact forces. The tool in combination with the safety module and the FTS has a weight of $2.2 \mathrm{~kg}$. The tool has several sharp edges, i.e. the radii of the tool are less than $0.2 \mathrm{~mm}$.

\section{B. Collision detection}

For detecting collisions and preventing to continue the motion after a collision, we use a disturbance observer introduced in [22], and extended in [23]. However, using the torque sensor measurements and the estimated generalized momentum $\boldsymbol{p}=\boldsymbol{M}(\boldsymbol{q}) \dot{\boldsymbol{q}}$ as internal state, we are able to determine the external torques. Conditioned by sensor noise and inaccuracies in the robot model we use a threshold so that the collision detection does not trigger during motion. In the case of a collision there are different possibilities to react. Closing brakes, switching to gravity-free mode or just stopping the robot by setting the current position to desired ones [23]. The latter is used in this paper, as it is often used in industrial HRC use cases.

\section{Comparison of forces with an active and an inactive safety module}

In order to compare the impact of a robot with a potentially unsafe tool and the developed safety module, we recorded the force data during impact. The forces are depicted in Fig. 4. For the impact area at the crash test dummy, we chose the forehead. The motion of the robot is an eight-DoF motion including the linear axis, and the type of the motion is chosen to be a possible standard motion in an industrial task. For the quantization of the robotic end-effector velocity, 

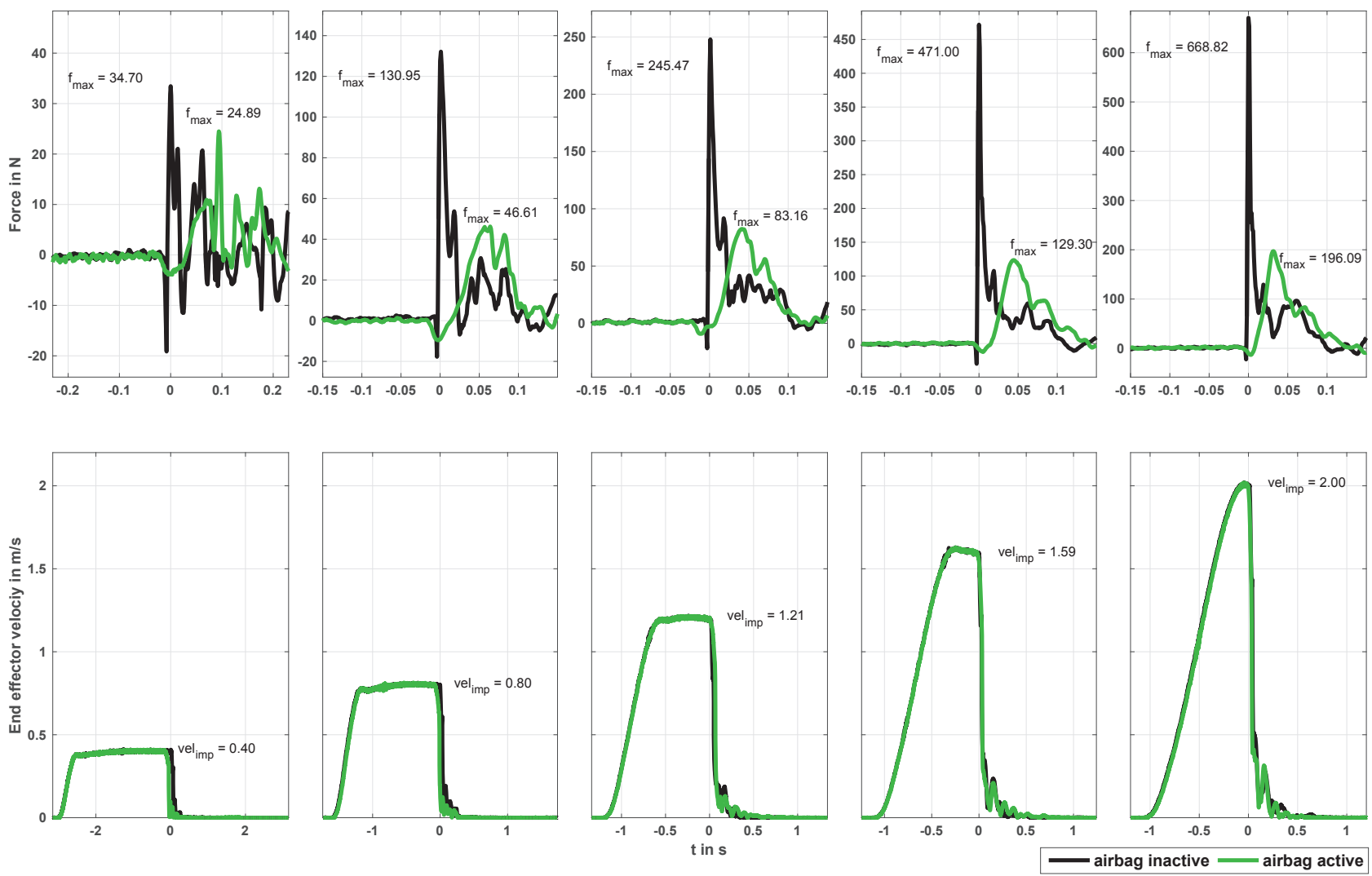

Fig. 4: Upper row: impact forces in $\mathrm{N}$ for a disabled (black line) and for an enabled (green line) safety module; bottom row: the associated velocity of the end-effector.

we selected steps of $0.4 \mathrm{~m} / \mathrm{s}$ with a velocity vector of $\dot{\boldsymbol{x}}=\left[\begin{array}{lllll}0.4 & 0.8 & 1.2 & 1.6 & 2\end{array}\right] \mathrm{m} / \mathrm{s}$, see Fig. 4 bottom row. At the time of the collision an abrupt drop of the velocity occurs. This depends on the impact forces and the collision strategy. In case of a detected collision, the robot stops immediately without closing the brakes, which of course has an effect on the resulting forces.

In the upper row of the figure the forces are shown over the time of the impact. The black solid line depicts the impact forces over time of the gripper hitting the human forehead. The gripper is uncovered and has no additional safety system. The green solid line depicts the same impact at the same impact velocity, but this time with the gripper covered by the safety module.

Using the safety module we reach a reduction of the maximum impact forces during slow motions $(0.4 \mathrm{~m} / \mathrm{s})$ of approximately $28.27 \%$, mid speed $(1.2 \mathrm{~ms}) 66.12 \%$ and fast motions $(2.0 \mathrm{~m} / \mathrm{s})$ up to $70.1 \%$. This is an essential reduction of impact forces. The force progression of an activated airbag exhibits a behavior of a spring-damper system, which can be observed in Fig. 4 green lines. The resulting contact forces depending on the end-effector velocity are depicted in Fig. 5] The slope of the contact forces regarding the activated airbag is substantially smaller.

Regarding ISO/TS 15066 the maximum quasi-static contact force for the middle of the forehead, which was the contact area at the dummy, is $130 \mathrm{~N}$. This implies that the robot is allowed to move with $0.8 \mathrm{~m} / \mathrm{s}$ in case of an

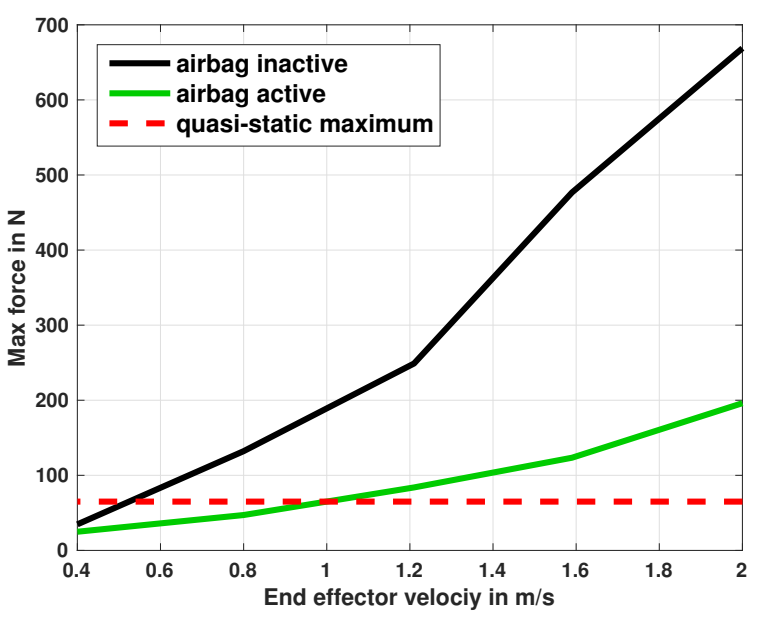

Fig. 5: Maximum force measurements during contact with the crash-test dummy depending on the velocity of the robot end-effector. The black solid line depicts the maximum absolute measured contact force with an inactive safety module. The green solid line depicts the maximum force with an active safety module.

inactive airbag. An activated airbag allows to move the robot with up to $1.6 \mathrm{~m} / \mathrm{s}$, leading to an substantial increase of the performance. Regarding the entire body, the maximum force 


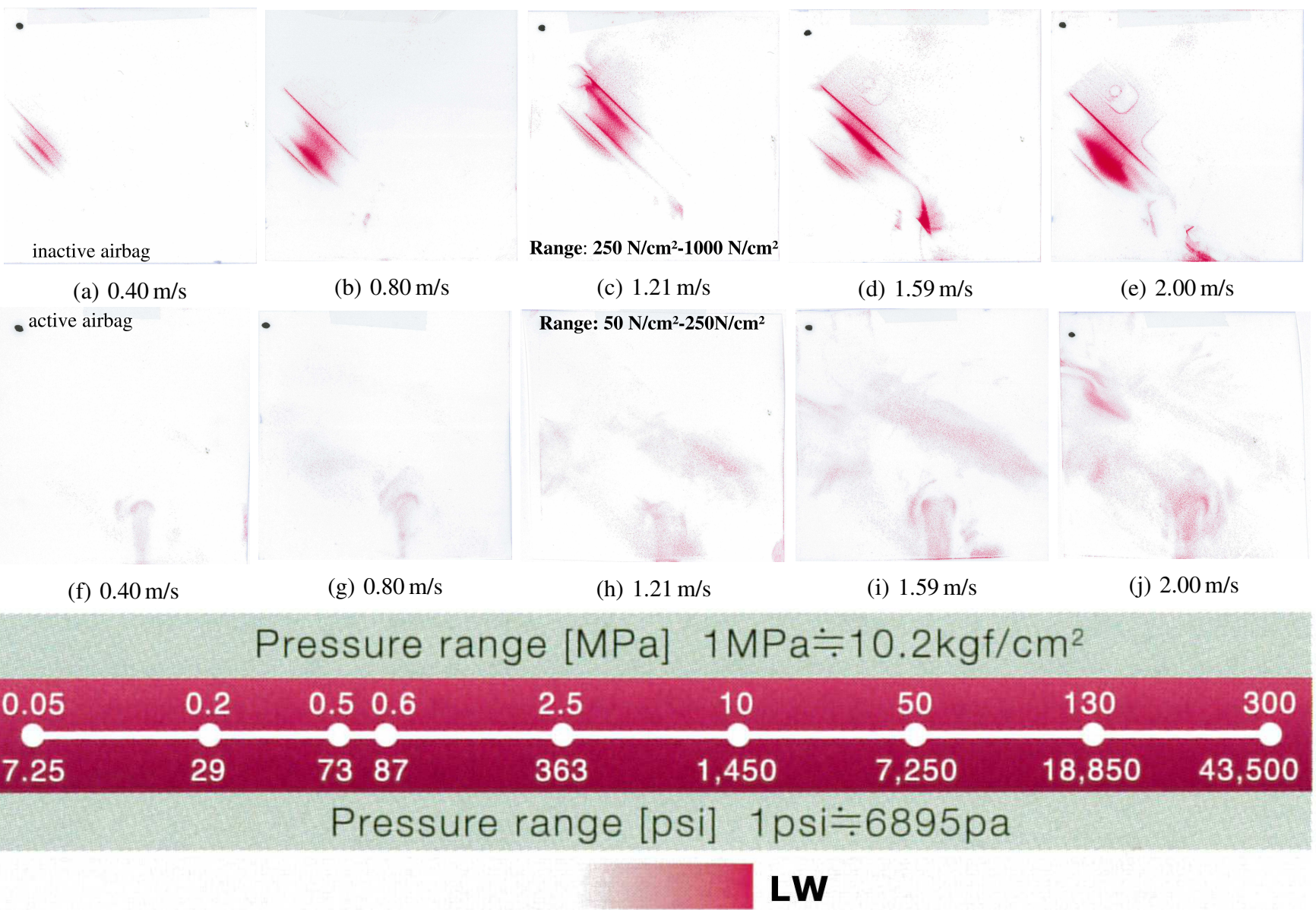

\section{LLW}

Fig. 6: Prescale pressure measurement films - Pressure distribution at the head of the crash-test dummy with deflated (upper row) and with inflated airbag safety system (lower row). For measuring the pressure distribution of the deflated airbag the Low pressure films (LW) with a pressure range between $250 \mathrm{~N} / \mathrm{cm}^{2}$ up to $1000 \mathrm{~N} / \mathrm{cm}^{2}$, and for the inflated airbag Super Low pressure films (LLW) with a pressure range between $50 \mathrm{~N} / \mathrm{cm}^{2}$ up to $250 \mathrm{~N} / \mathrm{cm}^{2}$ were used.

of quasi-statid 1 contact, reported in ISO/TS 15066 , is $65 \mathrm{~N}$ for the face, in particular the masticatory muscle. Using linear interpolation between $0.8 \mathrm{~m} / \mathrm{s}$ and $0.4 \mathrm{~m} / \mathrm{s}$ we are allowed to move the robot with approximately $0.53 \mathrm{~m} / \mathrm{s}$ for a deflated airbag. For the inflated airbag the respective interpolated velocity is $1.01 \mathrm{~m} / \mathrm{s}$, i. e. twice as large in comparison to an evacuated airbag.

\section{Experimental results with pressure film}

For measuring the peak pressure as described in ISO/TS 15066 we used Prescale pressure measurement films by Fujifilm. There are different types of measurement films, in particular they differ in their pressure range. To obtain a representative comparison to the data of ISO/TS 15066 which lies in the range of $110 \mathrm{~N} / \mathrm{cm}^{2}$ to $289 \mathrm{~N} / \mathrm{cm}^{2}$ we chose the super low pressure Prescale (LLW) with a range from $50 \mathrm{~N} / \mathrm{cm}^{2}$ up to $250 \mathrm{~N} / \mathrm{cm}^{2}$ and the low pressure Prescale (LW) with a range from $250 \mathrm{~N} / \mathrm{cm}^{2}$ up to $1000 \mathrm{~N} / \mathrm{cm}^{2}$. In

\footnotetext{
${ }^{1}$ Note, that there are no factor-values for transient contact in the ISO/TS 15066, i.e. the quasi-static limits matter
}

our experiments the films were attached to the middle of the forehead and the robot was moved until a collision occurred as described in Sec. III-C. It is important to notice that the experiments with the pressure film were done in parallel to the force experiments. It provides the possibility to compare maximum impact forces with the films, since both data are from the same runs.

In Fig. 6 the results of the experiments are shown. In the upper row the LW films were used for the experiments with the gripper and an inactive safety module. As one can see the emerging color of the film reaches the maximum at $0.8 \mathrm{~m} / \mathrm{s}$ and higher. Regarding the results of more than $1000 \mathrm{~N} / \mathrm{cm}^{2}$ for most of the pressure films of the deflated airbag the measured pressure peaks are much higher as suggested by ISO/TS 15066. It is not advisable to operate such a system without making safety arrangements. Moving the robot with less velocity makes the system inefficient and uneconomical. The second row in Fig. 6 depicts the results from a collision with an active safety module. It is important to notice that we used the LLW films with a range from $50 \mathrm{~N} / \mathrm{cm}^{2}$ up to 
$250 \mathrm{~N} / \mathrm{cm}^{2}$. As one can see, the results show an essential improvement of reducing the peak pressure with a maximum always below $250 \mathrm{~N} / \mathrm{cm}^{2}$.

If we assume that we have a full collaborative robotic work cell with a standard robotic gripper attached to the TCP as used in our experiments without any further safety systems, the limitation of the velocity is defined by the peak pressure and should definitely be less than $0.4 \mathrm{~m} / \mathrm{s}$. Moving the robot with an active safety module allows to set the velocity up to $1 \mathrm{~m} / \mathrm{s}$ regarding the maximum forces in a quasi-static contact. The results of the pressure measurement allow a velocity higher than $2 \mathrm{~m} / \mathrm{s}$. However, the maximum velocity is defined by the maximum forces of ISO/TS 15066. Regarding the reflected inertia, we used the streched out arm with a high reflectef inertia. Finally, with the proposed safety module we are able to move the robot with $1 \mathrm{~m} / \mathrm{s}$ given the assumption that the maximum velocity defined in ISO 10218 of $0.25 \mathrm{~m} / \mathrm{s}$ is suspended.

\section{CONCLUSION \& OUTLOOK}

This paper presented a new approach for safe physical human-robot interaction related to the danger of a robotic tool. We developed a safety module similar to the well known concept of airbags used in automobile industry for reducing consequences of a blunt impact. The difference to common airbags is the re-usability and that the airbag is inflated during every motion of the robot. We showed that a collaborative task with a robot can be performed much more efficiently using the safety module. We analyzed the force data of a collision with a crash-test dummy and the occurring pressure by using pressure measurement films. We found out that we can increase the velocity up to $1 \mathrm{~m} / \mathrm{s}$ while satisfying the requirements of ISO/TS 15066 with the presented experimental setup. Using the acceleration and deceleration time for inflating and deflating the airbag that no danger of injury can occur, we are able to perform an industrial task in an efficient and economic way.

Nevertheless, there is still the potential risk of pressure drop in the airbag during the manipulation of objects. Thus, we are going to analyze different sensor technologies in future studies in order to observe the status of the airbag. E.g., we will apply pressure sensors to observe unreasonably high or low pressure which reasons a malfunction of the airbag and requires to switch off the airbag and reduce the end-effector velocities. Furthermore, we will analyze the risk moving the end-effector to the central axis of the device.

\section{ACKNOWLEDGEMENT}

We thank Freek Stulp for proofreading previous versions of this document. This work has been partially funded by the Helmholtz Association as part of the project RACELab.

\section{REFERENCES}

[1] PILZ, "Safe camera system SafetyEYE," https://www.pilz.com/enINT/eshop/00106002207042/SafetyEYE-Safe-camera-system, 2016, [Online; accessed 13-September-2016].

[2] F. IFF, "Safe Human-Robot Interaction," http://www.iff.fraunhofer.de/ en/business-units/robotic-systems/research/human-robot-interaction. html, 2016, [Online; accessed 13-September-2016].
[3] A. Albu-Schäffer, S. Haddadin, C. Ott, A. Stemmer, T. Wimböck, and G. Hirzinger, "The dlr lightweight robot - lightweight design and soft robotics control concepts for robots in human environments," Industrial Robot Journal, vol. 34, no. 5, pp. 376-385, 2007.

[4] ISO, "Iso 10218-2:2011 robots and robotic devices - safety requirements for industrial robots - Part 2: Robot systems and integration," http://www.iso.org/iso/home/store/catalogue_tc/catalogue_detail. htm?csnumber=41571, 2016, [Online; accessed 13-September-2016].

[5] ISO/TS, "Iso/ts 15066:2016 robots and robotic devices - collaborative robots," http://www.iso.org/iso/catalogue_detail?csnumber=62996. 2016, [Online; accessed 13-September-2016].

[6] Y. Yamada, Y. Hirasawa, S. Huang, Y. Umetani, and K. Suita, "Humanrobot contact in the safeguarding space," IEEE/ASME Transactions on Mechatronics, vol. 2, no. 4, pp. 230-236, 1997.

[7] S. Haddadin, A. Albu-Schäffer, M. Frommberger, J. Rossmann, and G. Hirzinger, "The "DLR Crash Report": Towards a standard crashtesting protocol for robot safety - Part I: Results," International Conference on Robotics and Automation (ICRA), pp. 27 $\overline{2-279,2009 .}$

[8] ", "The DLR Crash Report": Towards a standard crash-testing protocol for robot safety - Part II: Discussions," International Conference on Robotics and Automation (ICRA), pp. 272-279, 2009.

[9] S. Haddadin, A. Albu-Schäffer, and G. Hirzinger, "The role of the robot mass and velocity in physical human-robot interaction - part i: Non-constrained blunt impacts," International Conference on Robotics and Automation (ICRA), pp. 1331-1338, 2008.

[10] S. Haddadin, A. Albu-Schäffer, M. Frommberger, and G. Hirzinger, "The role of the robot mass and velocity in physical human-robot interaction - part ii: Constrained blunt impacts," International Conference on Robotics and Automation (ICRA), pp. 1339-1345, 2008.

[11] S. Haddadin, S. Haddadin, A. Khoury, T. Rokahr, S. Parusel, R. Burgkart, A. Bicchi, and A. Albu-Schäffer, "A truly safely moving robot has to know what injury it may cause," IEEE/RSJ International Conference on Intelligent Robots and Systems (IROS), pp. 5406-5413, 2012.

[12] A. Bicchi and G. Tonietti, "Fast and soft arm tactics: Dealing with the safety-performance tredeoff in robot arms design and control," IEEE Robotics and Automation Magazine, vol. 11, no. 2, pp. 22-33, 2004.

[13] K. Ikuta, H. Ishii, and M. Nokata, "Safety evaluation method of design and control for human-care robots," The International Journal of Robotics Research, vol. 22, no. 5, pp. 281-298, 2003.

[14] M. P. Polverini, A. M. Zanchettin, and P. Rocco, "Real-time collision avoidance in human -robot interaction based on kineostatic safety field," IEEE/RSJ International Conference on Intelligent Robots and Systems (IROS), pp. 4136-4141, 2015.

[15] A. De Luca and F. Flacco, "Integrated control for phri: Collision avoidance, detection, reaction and collaboration," EEE RAS/EMBS International Conference on Biomedical Robotics and Biomechatronics, pp. 288-295, 2012.

[16] J. Mainprice and D. Berenson, "Human-robot collaborative manipulation planning using early prediction of human motion," IEEE/RSJ International Conference on Intelligent Robots and Systems (IROS), pp. 299-306, 2013.

[17] BOSCH, "APAS workstation - flexible product assistant," http:// www.bosch-apas.com/en/apas/start/bosch_apas.html, 2016, [Online; accessed 14-September-2016].

[18] B. Danube, "Airskin safety," http://www.bluedanuberobotics.com/? page_id=87, 2016, [Online; accessed 14-September-2016].

[19] L. Zeng and G. M. Bone, "Design of foam covering for robotic arms to ensure human safety," Electrical and Computer Engineering, pp. 1145-1150, 2008.

[20] Schunk, "Co-act gripper meets cobots," http://de.schunk.com/de_en/ co-act/, 2016, [Online; accessed 14-September-2016].

[21] FUJIFILM, "Pressure measurement film," http://www.fujifilmusa.com/ products/measurement-films/prescale/film/, 2016, [Online; accessed 13-September-2016].

[22] A. De Luca, S. Haddadin, A. Albu-Schäffer, and G. Hirzinger, "Collision detection and safe reaction with the DLR-III lightweight manipulator arm," IEEE/RSJ International Conference on Intelligent Robots and Systems (IROS), pp. 1623-1630, 2006.

[23] S. Haddadin, A. Albu-Schäffer, A. De Luca, and G. Hirzinger, "Collision detection and reaction: A contribution to safe ohysical humanrobot interaction," IEEE/RSJ International Conference on Intelligent Robots and Systems (IROS), pp. 3356-3363, 2008. 